Check for updates

Kent

Cite this as: $B M J 2021 ; 375: n 2411$ http://dx.doi.org/10.1136/bmj.n2411 Published: 01 October 2021

\title{
Vaccinating against covid and flu at same time is safe, study shows
}

\section{Jacqui Wise}

Administering an influenza vaccine at the same time as a second dose of a covid-19 vaccine produced no safety concerns and preserves the immune response to both vaccines, say researchers. ${ }^{1}$

The results from the ComFluCOV study have already been presented to the UK Joint Committee on Vaccination and Immunisation and fed into the committee's recent advice that the flu vaccine can be co-administered with a booster or third dose of a covid-19 vaccine. ${ }^{2}$

The study, funded by the National Institute for Health Research, has been published as a preprint and has not yet been peer reviewed.

A total of 679 volunteers were recruited across 12 NHS sites in England and Wales. They were all aged over 18 and had already received one dose of either the Pfizer-BioNTech or the Oxford-AstraZeneca vaccine and were waiting for their second dose. Two covid vaccines and three flu vaccines were tested, giving a total of six combinations.

The volunteers were randomly allocated to receive either a flu vaccine or a placebo saline injection in the opposite arm to their second covid-19 vaccine. Three weeks later the group who received placebo received the flu vaccine and vice versa. The participants also attended a third study visit to discuss any side effects they experienced and to give a blood sample.

The primary endpoint was one or more reported systemic reactions in the seven days after first trial vaccinations, with a difference of less than $25 \%$ considered to be acceptable. The most common side effects were pain around the injection site and fatigue. No significant difference was found in four of the six group combinations; in two combinations there was a slight increase in the number of people who reported at least one side effect when both covid-19 and flu vaccine were given together, but the reactions were mostly mild or moderate. The study also found that the immune responses to both the influenza and covid-19 vaccine were preserved when given together.

A strength of the study was that it did not exclude people who were pregnant, had severe uncontrolled medical problems, were immunocompromised, or aged 65 or over, so it was representative of the population who were most likely to receive both flu and covid vaccines. However, two of the groups had lower recruitment than planned, which was related to expiry dates of some flu vaccines and the timing of the roll out of specific covid-19 vaccines in the UK.

Rajeka Lazarus, a consultant in infectious diseases and microbiology at University Hospitals Bristol and Weston NHS Foundation Trust and the study's chief investigator, said, “This is a really positive step which could mean fewer appointments for those who require both vaccines, reducing the burden on those who have underlying health conditions and would usually be offered the influenza vaccine.” She told a Science Media Centre briefing that this would be a practical benefit to general practices as it would reduce the administration burden of making two appointments.

The JCVI guidance states that "where operationally expedient, covid-19 and influenza vaccines may be co-administered." However, it advises that the "covid-19 booster vaccine programme should not disrupt or delay deployment of the annual influenza vaccination programme." Practices should offer patients their covid and flu vaccines as soon as they are eligible rather than delaying while waiting for deliveries of either vaccine so as to administer them at the same time.

Lazarus R, Baos S, Cappel-Porter H, etal. The safety and immunogenicity of concomitant administration of COVID-19 vaccines (ChAdOx1 or BNT162b2 with seasonal influenza vaccines in adults: a phase IV, multicentre randomised controlled trial with blinding (ComFluCOV).https://ssrn.com/abstract $=3931758$.

2 JCVI statement regarding a COVID-19 booster vaccine programme for winter 2021 to 2022. Gov.UK. Sep 2021. https://www.gov.uk/government/publications/jcvi-statement-september-2021-covid-19-booster-vaccine-programme-for-winter-2021-to-2022/jcvi-statement-regarding-a-covid-19booster-vaccine-programme-for-winter-2021-to-2022.

This article is made freely available for use in accordance with BMJ's website terms and conditions for the duration of the covid-19 pandemic or until otherwise determined by BMJ. You may use, download and print the article for any lawful, non-commercial purpose (including text and data mining) provided that all copyright notices and trade marks are retained. 